$>$ Le calcium est un régulateur essentiel pour un grand nombre de fonctions cellulaires, et une perturbation de l'homéostasie calcique peut sévèrement troubler la physiologie de différents tissus. CASPI, STIMI, et ORAII codent pour des facteurs clés contrôlant les flux de calcium, et des mutations de ces gènes sont à l'origine de la myopathie à agrégats tubulaires et du syndrome de Stormorken. Ces deux maladies forment un continuum clinique regroupant faiblesse musculaire, myosis, thrombopénie, hyposplénisme, ichthyose, dyslexie et petite taille. <

La myopathie à agrégats tubulaires (TAM) est une maladie musculaire progressive caractérisée par une faiblesse musculaire, des crampes et myalgies, et les biopsies de personnes atteintes montrent typiquement des agencements denses de tubules membranaires [1]. Certains patients présentent des signes cliniques additionnels comme myosis, thrombocytopénie, hyposplénisme, ichthyose, dyslexie, et petite taille, et ce phénotype multi-systémique est appelé syndrome de Stormorken (STRMK) [2].

La myopathie à agrégats tubulaires et syndrome de Stormorken sont dus à des mutations hétérozygotes de STIMI [3-6] ou ORAII [6, 7], alors que des mutations hétérozygotes de CASOl ont été trouvées chez des patients avec un phénotype exclusivement musculaire $[8,9]$.

Les trois gènes codent des régulateurs clés de l'homéostasie calcique, et agissent de ce fait sur une multitude de voies cellulaires dépendantes du calcium, dont la contraction musculaire. La calséquestrine, codée par CASPl, joue un rôle majeur dans le stockage de calcium dans le réticulum, et participe activement au relargage du calcium vers le cytosol [10]. STIMl est un senseur réticulaire de calcium, et peut activer le canal calcique ORAll, situé dans la membrane plasmique, afin d'induire l'entrée de calcium extracellulaire à travers un mécanisme appelé SOCE (store-operated calcium entry) [11-13]. Le SOCE existe dans tout type de cellule, ce qui sous-tend le large spectre phénoty-

\section{La myopathie à agrégats tubulaires et le syndrome de Stormorken}

Johann Böhm ${ }^{1-4}$, Jocelyn Laporte ${ }^{1-4}$

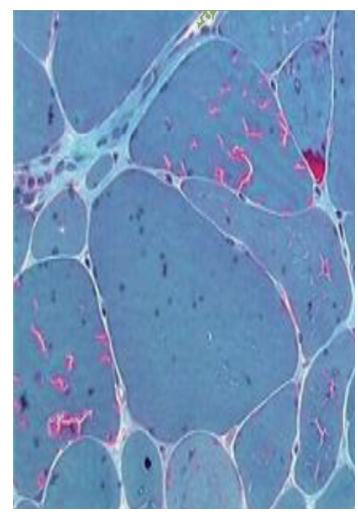

${ }^{1}$ Institut de Génétique et de Biologie Moléculaire et Cellulaire (IGBMC), IIlkirch, France.

${ }^{2}$ Centre National de la Recherche Scientifique (CNRS), UMR7104, Illkirch, France.

${ }^{3}$ Institut National de la Santé et de la Recherche Médicale (INSERM), U1258, IIIkirch, France. ${ }^{4}$ Université de Strasbourg, Illkirch, France.

pique chez les patients TAM/STRMK avec mutations de STIMI ou ORAII.

johann@igbmc.fr

Des analyses fonctionnelles ont démontré qu'il s'agit de mutations gain-de-fonction provoquant une sur-activation du SOCE et une entrée excessive de calcium $[3,6,7]$.

Cet article vise à résumer les caractéristiques cliniques et histologiques de la myopathie à agrégats tubulaires et du syndrome de Stormorken, et à analyser la corrélation entre génotype et phénotype avec une attention particulière pour les pathomécanismes entraînant le dysfonctionnement musculaire.

\section{Présentation clinique et histologique de la myopathie à agrégats tubulaires et du syndrome de Stormorken}

Au niveau clinique, la myopathie à agrégats tubulaires se fait surtout remarquer par des crampes, des myalgies ainsi qu'une faiblesse musculaire progressive touchant d'abord les muscles proximaux des membres inférieurs [1]. Le niveau de créatine kinase dans le sérum est souvent dix fois supérieur à la normale, et certains patients développent des rétractions dans les bras et les jambes, ainsi que des troubles de l'oculomotricité $[3,7,9,14]$. Des symptômes multi-systémiques comme une thrombocytopénie, un myosis, une ichthyose, une dyslexie, ou une petite taille peuvent être plus ou moins prononcés, et la totalité de cette présentation clinique est diagnostiqué en tant que syndrome de Stormorken [2, 7, 15-19]. De manière générale, TAM et STRMK forment un continuum clinique avec des patients TAM qui 


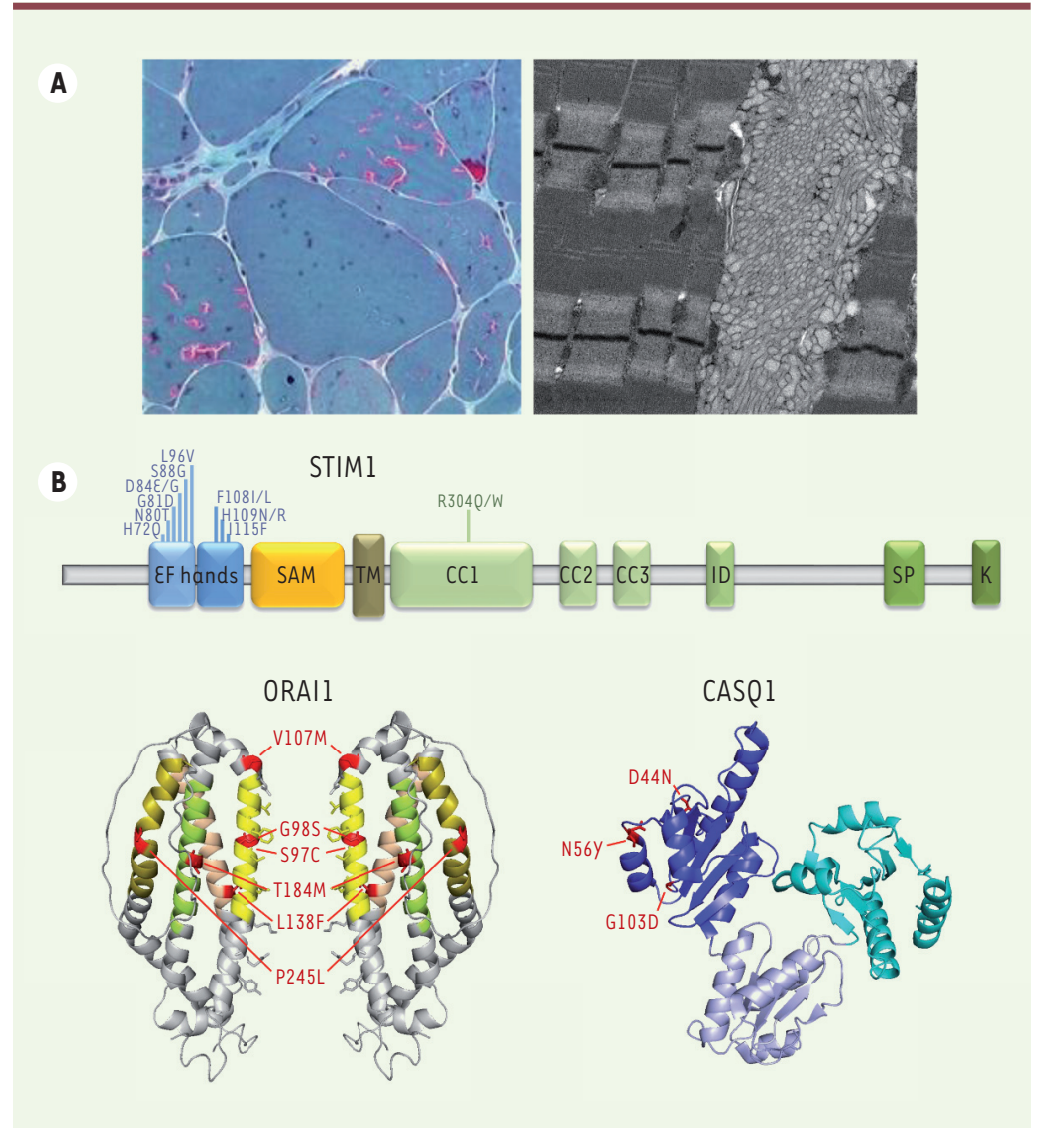

Figure 1. A. Coloration de trichrome de Gomori d'une biopsie musculaire d'un patient TAM/STRMK montrant une inégalité de taille de fibres, ainsi que des accumulations basophiles en rouge. La microscopie électronique dévoile que ces accumulations correspondent à des agencements réguliers de tubules membranaires. B. STIMl est une protéine transmembranaire du réticulum avec les domaines EF et SAM formant la partie luminale, un domaine transmembranaire (TM), et des domaines coiled-coil (CCl, CC2, CC3) contenant le SOAR (STIMI-ORAIl activating domain), un domaine inhibiteur (ID), et des régions riches en sérine/proline (SP) et lysine (K) dans la partie cytosolique. La majorité des mutations fauxsens s'accumule dans les domaines EF liant le calcium. ORAll est un canal calcique de la membrane plasmique, et chaque monmère est composé de quatre domaines transmembranaires (hélices alpha colorées) avec le domaine Ml formant le puit. La position des mutations est marquée en rouge. La calséquestrine (CASPl) est composée de trois domaines thiorédoxines globulaires avec un noyau hydrophobe et une surface d'acides aminés négativement chargés pour lier le calcium. Les mutations sont marquées en rouge à l'exception d'I385T, qui n'apparaît pas sur le modèle de la structure résolue de 383 acides aminés.

peuvent montrer un ou plusieurs signes du syndrome de Stormorken, et certains patients avec syndrome de Stormorken qui présentent surtout une faiblesse musculaire [16]. À noter qu'une troisième maladie fait également partie de ce continuum: le syndrome plaquettaire de York (YPS) a initialement été décrit comme maladie hématologique [20], mais des examens cliniques complets ont révélé des symptômes musculaires et non-musculaires correspondant à la TAM et au syndrome de Stormorken [16].

Les biopsies musculaires de patients TAM/STRMK montrent des accumulations basophiles centrales ou sous-sarcolemmiques surtout dans les fibres de type II. Ces accumulations apparaissent en rouge après coloration de trichrome de Gomori, et en bleu après coloration NADHTR. A l'échelle ultra-structurale, ces accumulations correspondent à des agencements réguliers de tubules membranaires d'un diamètre de 20 à $200 \mathrm{~nm}$ [1, 21] (Figure 1A). À part ce trait caractéristique, d'autres signes histopathologiques comme une variabilité de taille de fibres, des noyaux internalisés, une prédominance de fibres de type I, ou une atrophie des fibres de type II ont également été décrits chez de patients avec TAM/STRMK $[3,7,9,14,15,17,18,22,23]$.

\section{Origine des agrégats tubulaires et présence dans d'autres myopathies}

Les agrégats tubulaires ne sont pas réactifs aux colorations histologiques SDH et COX, ce qui exclut une origine mitochondriale [1, 24].
Des analyses sur coupes musculaires par immunofluorescence ont démontré que les agrégats tubulaires contiennent différentes protéines réticulaires comme le senseur de calcium STIMl, les pompes calciques SERCAl et SERCA2, le canal calcique RyR1, et le tampon de calcium calséquestrine $[1,3,7,9,15,23]$. II est donc probable qu'il s'agit de structures issues du réticulum sarcoplasmique. Cependant, le mécanisme de formation des agrégats tubulaires n'est pas connu. II est possible que l'entrée excessive de calcium dans les fibres musculaires de patients avec TAM ou syndrome de Stormorken induise une dilatation du réticulum en tant que compartiment principal de stockage de calcium, et que des replis membranaires dus à cette dilatation représentent une première étape dans la formation des agrégats tubulaires.

Il est important de noter que les agrégats tubulaires sont aussi trouvés en tant que traits secondaires dans d'autres maladies musculaires transmises ou acquises telles que l'hyperthermie maligne, la paralysie périodique hypokaliémique, ou les myopathies métaboliques, inflammatoires et éthyliques [25-27]. Les agrégats tubulaires sont également présents dans les syndromes myasthéniques dus à des mutations de DPAGT1, ALG2, ou encore GFPTI [28]. 


\section{Gènes et protéines impliqués}

La majorité des patients TAM/STRMK avec diagnostic moléculaire porte des mutations de STIMI, et les mutations d'ORAII sont moins fréquentes. II s'agit toujours de mutations hétérozygotes avec gain-de fonction, alors que des mutations récessives perte-de-fonction dans ces deux gènes ont été associées à une immunodéficience sévère, caractérisée par des infections récurrentes et chroniques, une autoimmunité, une dysplasie ectodermale, et une hypotonie musculaire [29-31]. CASPl a été récemment identifié comme troisième gène de TAM chez des patients qui ne manifestent aucun des signes non-musculaires du syndrome de Stormorken [8,9].

STIMl est une protéine transmembranaire avec une partie $\mathrm{N}$-terminale dans la lumière du réticulum, et une partie $\mathrm{C}$-terminale cytosolique. La partie luminale contient deux domaines $\varepsilon F$ capables de lier le calcium, et un domaine SAM (sterile $\alpha$-motif), resserré aux domaines EF à l'état inactif [32]. La déplétion des réserves calciques et le détachement du calcium des domaines $E F$ induit un changement de conformation et un dépliement de STIMl, qui va ensuite di- et multimériser [32]. La partie cytosolique de STIMI est constituée de différents domaines et régions, dont notamment le domaine SOAR (STIMI-ORAll activating domain) permettant l'interaction avec le canal calcique ORAll et son activation, et une hélice inhibitrice régulant cette interaction [11, 33, 34].

ORAll fait partie des canaux CRAC (calcium release-activated calcium channels), qui se distinguent par une forte sélectivité pour le calcium, et une faible conductance [35]. ORAll est activé suite à la déplétion des réserves calciques par STIMl et le SOCE, et inactivé par des concentrations élevées de calcium cytosolique (CDI ; calciumdependent inactivation). Ce canal de la membrane plasmique est composé de tétramères ou hexamères qui forment des anneaux concentriques autour d'un puit central [36-38]. Chaque sous-unité contient quatre domaines transmembranaires ( $M l$ à $M 4$ ), avec $M l$ formant le puit, et M2 à M4 les anneaux [39]. L'ouverture du canal est régulé par I'interaction des parties $\mathrm{N}$ - et C-terminales avec STIMI [40].

Contrairement à l'expression ubiquitaire de STIMI et ORAIl, la calséquestrine est uniquement retrouvée dans le muscle squelettique, et notamment dans les fibres rapides de type II [41]. La calséquestrine est une protéine globulaire avec noyau hydrophobe et une surface exposant des acides aminés acides permettant la liaison électrostatique du calcium [42]. Elle participe activement au relargage de calcium vers le cytosol dans un complexe quartenaire avec RyRl, la junctine, et la triadine [10]. Elle est monomérique à faible concentration calcique, et les monomères de calséquestrine séquestrent STIMl et agissent ainsi en tant que régulateurs négatifs du SOCE [43]. L'entrée de calcium dans le réticulum induit la di- et polymérisation de la calséquestrine, ce qui augmente sa capacité de liaison de calcium [44-46].

\section{Corrélation génotype/phénotype}

À présent, 14 mutations différentes de STIMI ont été rapportées chez des patients TAM/STRMK, dont 12 mutations des domaines EF (H72P, N80T, G81D, D84E, D84G, S88G, L96V, F108I, F108L, H109N, H109R,
I115F) $[3,14,17,18,23]$, et deux mutations de la partie cytosolique (R3040 et R304W, Figure IB) $[4-6,16$, 23, 47]. II s'agit exclusivement de mutations faux-sens touchant des acides aminés conservés. La majorité de ces mutations est unique; N80T, G81D, et H109N ont été trouvées deux fois, et $\mathrm{H} 109 \mathrm{R}$ et $1115 \mathrm{~F}$ trois fois. La mutation R304W dans la partie cytosolique est la plus récurrente avec 12 familles connues et non-apparentées. L'autre mutation cytosolique touchant le même acide aminé, R304Q, a été rapportée dans une seule famille [23]. Il existe une corrélation partielle entre le génotype et le phénotype. La mutation récurrente R304W a principalement été trouvée chez des patients avec phénotype multi-systémique de syndrome de Stormorken $[4-6,23,47]$, et seuls quelques patients ont principalement une gêne musculaire [16]. À l'inverse, les patients avec mutation des domaines $\varepsilon F$ présentent surtout un phénotype musculaire et peu ou pas de symptômes non-musculaires. Le phénotype du muscle squelettique se traduit par une faiblesse musculaire ou une myalgie comme signe primaire et majeur $[3,14$, $16-18,22,23]$, et cela ne dépend pas uniquement de I'acide aminé affecté. La substitution du résidu F108 en isoleucine cause une faiblesse musculaire proximale dès l'enfance dans une famille, et le changement en leucine est associé à une myalgie à début tardif dans une autre famille [14]. Il existe même une variabilité clinique pour la même mutation. Une famille avec la mutation H109N était décrite avec fatigabilité postexercice, et une autre famille avec la même mutation montrait une faiblesse musculaire et des rétractions des membres inférieurs [3, 14].

Les mutations d'ORAI sont moins fréquentes avec sept familles TAM/STRMK rapportées à présent $[6,7,15$, 19]. Toutes les mutations touchent des acides aminés conservés dans les domaines transmembranaires: les faux-sens S97C, G98S, et V107M ont été trouvées dans le domaine transmembranaire $\mathrm{Ml}$ constituant le puit, et L138F, T184M, et P245L dans les domaines formant les anneaux concentriques (Figure 1B). G98S est la seule mutation rapportée deux fois, et les deux familles manifestent un phénotype sévère avec faiblesse musculaire marquée et rétractions dès l'enfance $[7,15]$. $\varepsilon n$ revanche, la famille avec la mutation voisine S97C était cliniquement plus modérée avec crampes et faiblesse musculaire à début tardif [19]. Généralement, on observe des crampes et une raideur musculaire surtout chez les patients ORAll $[6,7,15,19,48]$, et moins chez les patients STIMI. Une autre différence concerne les symptômes non-musculaires, qui sont globalement moins prononcés chez les patients ORAll, et certains signes du syndrome de Stormorken comme la thrombo- 
cytopénie, l'asplénie, ou la petite taille n'ont jamais été rapportés. La faiblesse musculaire est plutôt diffuse en cas de mutation d'ORAll, et souvent proximale pour STIMI. Des études d'IRM et de tomographie des membres inférieurs de patients ORAll ont démontré une atrophie symétrique et des infiltrations graisseuses dans les parties postérieures et médiales de la cuisse, et de la partie postérieure du bas de la jambe avec implication particulière des muscles gastrocnémiens $[7$, 15, 19]. Les patients STIMI montrent une atrophie et des infiltrations graisseuses dans la cuisse et le bas de jambe à l'exception de quelques muscles comme le tibial antérieur [49]. À noter que le muscle long fléchisseur de l'hallux, rarement affecté dans les maladies musculaires, est atteint chez les patients avec mutations de STIMI et ORAII [19, 49], ce qui pourrait représenter un signe distinctif entre la myopathie à agrégats tubulaires et le syndrome de Stormorken.

CASPl a récemment été identifié en tant que troisième gène TAM, et quatre mutations hétérozygotes différentes ont été décrites: D44N, N56Y, G103D, et I385T (Figure 1B) [8,9]. Le phénotype des patients est modéré par rapport aux patients STIMI et ORAII, et se limite à une faiblesse musculaire des membres inférieurs peu progressive et à début tardif, ainsi qu'à des myalgies et une fatigue post-exercice $[8,9$, 50]. Ceci est en accord avec l'expression exclusivement musculaire de CASP1 [41], qu'on trouve surtout dans les fibres de types II. Une autre mutation faux-sens dans CASPI (D244G) a été préalablement associée à la myopathie vacuolaire, qui diffère de la myopathie à agrégats tubulaires au niveau clinique et histologique [51].

\section{Pathomécanismes des mutations STIM1/ORAI1/CASP1}

Dans des cellules transfectées, STIMI a une localisation réticulaire diffuse, alors que l'expression de constructions avec mutations des domaines $\varepsilon F$ induit l'oligomérisation constitutive de la protéine $[3,14$, $17,18,22]$. Ceci suggère que les mutations du domaine $\varepsilon F$ impactent directement ou indirectement la coordination du calcium ou déstabilisent l'interaction entre les domaines EF et SAM, et provoquent ainsi le dépliement et l'oligomérisation de STIMl, et en conséquence l'activation du canal calcique ORAIl sans déplétion préalable des réserves calciques. En effet, des analyses ratiométriques sur myoblastes de patients ont révélé un niveau calcique basal élevé et une entrée excessive de calcium extracellulaire sans activation du SOCE [3, 18]. La mutation cytosolique R304W a le même effet dans les cellules transfectées et dans les myoblastes de patients $[4-6,23]$, mais le pathomécanisme est différent. Une étude récente a démontré que cette mutation induit une élongation hélicale dans le domaine cytosolique de STIMI provoquant l'exposition du domaine SOAR et l'activation d'ORAII [52]. Cette même étude a révélé un effet moins délétère de la mutation $R 3040$, ce qui va de pair avec un phénotype plus modéré des patients respectifs. L'effet pléiotropique de la mutation $\mathrm{R} 304 \mathrm{~W}$ peut s'expliquer par une répercussion particulière sur l'inactivation d'ORAIl calcium-dépendante (CDI), comme démontré par des études électrophysiologiques [6]. Comparée aux mutations des domaines $\varepsilon F$, la mutation R304W provoque une entrée calcique prolongée en conséquence d'une répression plus intense du CDI [6], et c'est ce surplus de calcium dans différents cellules et tissus qui serait à l'origine de symptômes multi-systémiques du syndrome de Stormorken.

L'expression exogène des mutants ORAll induit une augmentation du niveau calcique basal et une entrée excessive de calcium après activation du SOCE $[7,15$, 19], et des résultats similaires ont été obtenus dans des myotubes de patients [15, 19]. Des études dans des cellules murines sans Stiml et son paralogue Stim2 (Stim1 $1^{-/-} /$Stim2 $2^{-/-}$) ont été effectuées par la suite afin de déterminer si l'abondance de calcium était le résultat d'une perméabilité accrue ou d'une activation anormale du canal [7]. Une entrée constante d'ions était uniquement observée dans des cellules exprimant ORAIl avec des mutations du domaine Ml (G98S et V107M), alors qu'ORAll avec des mutations du domaine M3 (T184M) était indiscernable du contrôle. En revanche, les trois mutations induisaient bien une entrée excessive de calcium dans des cellules Stim1 $1^{-/-} /$Stim2 $^{-/-}$co-exprimant ORAIl et STIMI. Ces résultats indiquent un pathomécanisme différent en fonction des mutations. Les mutations touchant des acides aminés du puit génèrent un canal perméable indépendamment de STIMl, alors que des mutations des anneaux concentriques causent une activation anormale du canal via STIMl et le SOCE $[6,7]$. Des études dans cellules transfectées et sur protéines recombinantes ont montré que les mutations de CASPI entravaient la dynamique de polymérisation et dépolymérisation de la calséquestrine [8, 9]. Les mutants monomérisaient moins que le contrôle après déplétion des réserves calciques, et produisaient moins de grands polymères à des concentrations calciques croissantes. Ces observations suggèrent un double effet pathogénique des mutations de CASP1. Dans ce modèle, il y aurait moins de monomères de calséquestrine pour séquestrer STIMl et inhiber le SOCE, et moins de polymères de calséquestrine pour assurer le stockage de calcium dans le réticulum. En effet, le traitement de fibres musculaires isolées à la caféine a confirmé une capacité de stockage de calcium diminué dans le réticulum [8]. Contrairement aux mutations TAM, le mutant D244G, auparavant associé à la myopathie vacuolaire [51], produit des grands polymères insolubles de calséquestrine. Ceci suggère que les mutations de CASPl peuvent être à l'origine de deux myopathies différentes, et que ces deux maladies impliquent un pathomécanisme différent $[8,9]$.

\section{Résumé et directions futures}

Pour résumer, des mutations hetérozygotes gain-defonction dans STIMI et ORAII induisent une entrée excessive de calcium et causent la myopathie à agrégats tubulaires et le syndrome de Stormorken [3-7], 
tandis que les mutations hétérozygotes de CASQ1 promeuvent l'entrée de calcium à travers un impact sur la polymérisation et dépolymérisation de la calséquestrine $[8,9]$. À l'inverse, des mutations récessives perte-de-fonction de STIMI et ORAII suppriment l'entrée de calcium et entraînent une immunodéficience [29-31]. En conclusion, TAM et STRMK sont causés par un SOCE sur-actif, et l'immunodéficience par un SOCE sous-actif.

La recherche ultérieure sur la myopathie à agrégats tubulaires et le syndrome de Stormorken devra aborder des questions fondamentales sur la physiopathologie de ces maladies avec une attention particulière aux effets en aval de l'entrée excessive de calcium afin de déterminer la séquence des événements qui conduisent au dysfonctionnement musculaire et aux anomalies dans d'autres tissus. Même si l'excès de calcium est indubitablement à l'origine des phénotypes majeurs, il reste à déterminer si un déséquilibre potentiel d'autres ions contribue au développement de la maladie. II est par exemple possible que les mutations d'ORAIl impactent sur la sélectivité ionique du canal, et que la sur-activation de STIMl due à des mutations influence d'autres canaux. Il est en effet connu que STIMl régule les canaux TRP (transient potential receptor), qui sont bien moins sélectifs pour le calcium [53].

II n'y a actuellement pas de traitement pour la myopathie à agrégats tubulaires et le syndrome de Stormorken. Néanmoins, le déséquilibre de calcium est susceptible à la manipulation par des agents pharmacologiques, et la réduction de l'entrée excessive de calcium à travers des molécules inhibant le canal calcique ORAIl pourraient représenter une approche thérapeutique adaptée. $\diamond$

\section{SUMMARY}

\section{Tubular aggregate myopathy and Stormorken syndrome}

Calcium $\left(\mathrm{Ca}^{2+}\right)$ is an essential regulator for a large number of cellular functions in various tissues and organs, and small disturbances of $\mathrm{Ca}^{2+}$ homeostasis can severely compromise normal physiology. Intracellular $\mathrm{Ca}^{2+}$ balance is mainly controlled by the reticular $\mathrm{Ca}^{2+}$ sensor STIMI and the plasma membrane $\mathrm{Ca}^{2+}$ channel ORAIl through a mechanism known as store-operated $\mathrm{Ca}^{2+}$ entry (SOCE). Gain-of-function mutations in STIMI or ORAIl cause excessive extracellular $\mathrm{Ca}^{2+}$ influx, resulting in tubular aggregate myopathy (TAM) and Stormorken syndrome (STRMK). Both disorders are spectra of the same disease and involve muscle weakness, miosis, thrombocytopenia, hyposplenism, ichthyosis, dyslexia, and short stature. Here we summarize the clinical and histological characteristics of both disorders, provide an overview on the genetic causes, and recapitulate the current knowledge on the pathomechanisms leading to the multi-systemic phenotype of tubular aggregate myopathy and Stormorken syndrome. $\diamond$

\section{REMERCIEMENTS}

Les auteurs souhaitent remercier Raphaël Schneider pour son aide pour la réalisation de la figure.

\section{LIENS D'INTÉRÊT}

Les auteurs déclarent n'avoir aucun lien d'intérêt concernant les données publiées dans cet article.

\section{RéFÉRENCES}

1. Chevessier F, Bauche-Godard S, Leroy JP, et al. The origin of tubular aggregates in human myopathies. J Pathol 2005 ; 207 : 313-23.

2. Stormorken $H$, Sjaastad 0 , Langslet $A$, et al. A new syndrome: thrombocytopathia, muscle fatigue, asplenia, miosis, migraine, dyslexia and ichthyosis. Clin Genet $1985 ; 28$ : 367-74.

3. Bohm J, Chevessier F, Maues De Paula A, et al. Constitutive activation of the calcium sensor STIMl causes tubular-aggregate myopathy. Am J Hum Genet $2013 ; 92: 271-8$.

4. Misceo D, Holmgren A, Louch WE, et al. A dominant STIMl mutation causes Stormorken syndrome. Hum Mutat 2014 ; 35 : 556-64.

5. Morin G, Bruechle NO, Singh AR, et al. Gain-of-function mutation in STIMl (P.R304W) is associated with Stormorken syndrome. Hum Mutat 2014 ; 35 : 1221-32.

6. Nesin V, Wiley G, Kousi M, et al. Activating mutations in STIMl and ORAII cause overlapping syndromes of tubular myopathy and congenital miosis. Proc Natl Acad Sci USA 2014 ; 111 : 4197-202.

7. Bohm J, Bulla M, Urquhart JE, et al. ORAll mutations with distinct channel gating defects in tubular aggregate myopathy. Hum Mutat 2017 ; 38 : 426-38.

8. Barone V, Del Re V, Gamberucci A, et al. Identification and characterization of three novel mutations in the CASPl gene in four patients with tubular aggregate myopathy. Hum Mutat 2017 ; 38 : 1761-73.

9. Bohm J, Lornage X, Chevessier F, et al. CASQl mutations impair calsequestrin polymerization and cause tubular aggregate myopathy. Acta Neuropathol 2018 ; $135 ; 149-51$.

10. Lee KW, Maeng JS, Choi JY, et al. Role of Junctin protein interactions in cellular dynamics of calsequestrin polymer upon calcium perturbation. J Biol Chem $2012 ; 287: 1679-87$.

11. Park CY, Hoover PJ, Mullins FM, et al. STIMI clusters and activates CRAC channels via direct binding of a cytosolic domain to Orail. Cell 2009; 136 : 876-90.

12. Luik RM, Wu MM, Buchanan J, et al. The elementary unit of store-operated $\mathrm{Ca}^{2+}$ entry: local activation of CRAC channels by STIMI at $\varepsilon R-p l a s m a$ membrane junctions. J Cell Biol $2006 ; 174: 815-25$.

13. Stathopulos PB, Zheng L, Li GY, et al. Structural and mechanistic insights into STIMI-mediated initiation of store-operated calcium entry. Cell 2008 ; $135: 110-22$.

14. Bohm J, Chevessier F, Koch C, et al. Clinical, histological and genetic characterisation of patients with tubular aggregate myopathy caused by mutations in STIMl.J Med Genet $2014 ; 51: 824-33$.

15. Endo Y, Noguchi S, Hara Y, et al. Dominant mutations in ORAll cause tubular aggregate myopathy with hypocalcemia via constitutive activation of storeoperated $\mathrm{Ca}^{2+}$ channels. Hum Mol Genet 2015 ; $24: 637-48$.

16. Markello T, Chen D, Kwan Jy, et al. York platelet syndrome is a CRAC channelopathy due to gain-of-function mutations in STIMl. Mol Genet Metab 2015; 114 : 474-82.

17. Noury JB, Bohm J, Peche GA, et al. Tubular aggregate myopathy with features of Stormorken disease due to a new STIMl mutation. Neuromuscul Disord $2017 ; 27: 78-82$

18. Walter MC, Rossius M, Zitzelsberger M, et al. 50 years to diagnosis: Autosomal dominant tubular aggregate myopathy caused by a novel STIMI mutation. Neuromuscul Disord $2015 ; 25$ : 577-84.

19. Garibaldi M, Fattori F, Riva B, et al. A novel gain-of-function mutation in ORAIl causes late-onset tubular aggregate myopathy and congenital miosis. Clin Genet $2017 ; 91: 780-6$.

20. White JG. Giant electron-dense chains, clusters and granules in megakaryocytes and platelets with normal dense bodies: an inherited thrombocytopenic disorder. Platelets $2003 ; 14: 109-21$.

21. Muller HD, Vielhaber S, Brunn A, et al. Dominantly inherited myopathy with novel tubular aggregates containing 1-21 tubulofilamentous structures. Acta Neuropathol $2001 ; 102: 27-35$.

22. Hedberg C, Niceta M, Fattori F, et al. Childhood onset tubular aggregate myopathy associated with de novo STIMI mutations. J Neurol $2014 ; 261$ $870-6$.

23. Harris $\varepsilon$, Burki U, Marini-Bettolo C, et al. Complex phenotypes associated with STIMl mutations in both coiled coil and EF-hand domains. Neuromuscul Disord $2017 ; 27: 861-72$.

24. Chevessier F, Marty I, Paturneau-Jouas M, et al. Tubular aggregates are from whole sarcoplasmic reticulum origin: alterations in calcium binding protein expression in mouse skeletal muscle during aging. Neuromuscul Disord $2001 ; 14: 208-16$. 


\section{RéFÉRENCES}

25. Schiaffino $S$. Tubular aggregates in skeletal muscle: just a special type of protein aggregates? Neuromuscul Disord $2012 ; 22$ : 199-207.

26. Goebel HH. When tubules aggregate. Neuromuscul Disord $2012 ; 22$ : 208-10.

27. Engel WK, Bishop DW, Cunningham GG. Tubular aggregates in type II muscle fibers: ultrastructura and histochemical correlation. J Ultrastruct Res $1970 ; 31: 507-25$.

28. Engel AG, Shen XM, Selcen D, et al. Congenital myasthenic syndromes: pathogenesis, diagnosis, and treatment. Lancet Neurol 2015; $14: 420-34$.

29. Feske $S$, Gwack $Y$, Prakriya $M$, et al. A mutation in Orail causes immune deficiency by abrogating CRAC channel function. Nature $2006 ; 441: 179-85$.

30. Picard C, McCarl CA, Papolos A, et al. STIMl mutation associated with a syndrome of immunodeficiency and autoimmunity. N Engl J Med 2009 ; 360 : 1971-80.

31. Lacruz RS, Feske S. Diseases caused by mutations in ORAIl and STIMI, Ann NY Acad Sci 2015 ; $1356: 45-79$.

32. Stathopulos PB, Li GY, Plevin MJ, et al. Stored $\mathrm{Ca}^{2+}$ depletion-induced oligomerization of stromal interaction molecule l (STIMI) via the EF-SAM region: an initiation mechanism for capacitive $\mathrm{Ca}^{2+}$ entry. J Biol Chem 2006 ; 281 : 35855-62.

33. Yuan JP, Zeng W, Dorwart MR, et al. SOAR and the polybasic STIMI domains gate and regulate Orai channels. Nat Cell Biol $2009 ; 11$ : 337-43.

34. Kawasaki T, Lange I, Feske S. A minimal regulatory domain in the $C$ terminus of STIMI binds to and activates ORAIl CRAC channels. Biochem Biophys Res Commun 2009 ; 385 : 49-54.

35. Prakriya M. The molecular physiology of CRAC channels. Immunol Rev $2009 ; 231: 88-98$.

36. Cai X, Zhou Y, Nwokonko RM, et al. The Orail store-operated calcium channel functions as a hexamer. J Biol Chem $2016 ; 291: 25764-75$.

37. Hou X, Pedi L, Diver MM, et al. Crystal structure of the calcium release-activated calcium channe Orai. Science $2012 ; 338: 1308-13$.

38. Thompson JL, Shuttleworth TJ. How many Orai's does it take to make a CRAC channel? Sci Rep $2013 ; 3: 1961$.

39. Vig M, Beck A, Billingsley JM, et al. CRACMl multimers form the ion-selective pore of the CRAC channel. Curr Biol $2006 ; 16: 2073-9$.

40. Zheng $\mathrm{H}$, Zhou MH, Hu C, et al. Differential roles of the $\mathrm{C}$ and $\mathrm{N}$ termini of Orail protein in interacting with stromal interaction molecule 1 (STIMI) for $\mathrm{Ca}^{2+}$ release-activated $\mathrm{Ca}^{2+}$ (CRAC) channel activation. J Biol Chem 2013; $288: 11263-72$.

41. Schiaffino S, Reggiani C. Fiber types in mammalian skeletal muscles. Physiol Rev $2011 ; 91$ : $1447-531$.
42. Wang $\mathrm{S}$, Trumble WR, Liao $\mathrm{H}$, et al. Crystal structure of calsequestrin from rabbit skeletal muscle sarcoplasmic reticulum. Nat Struct Biol $1998 ; 5$ : 476-83.

43. Wang L, Zhang L, Li S, et al. Retrograde regulation of STIMl-Orail interaction and store-operated $\mathrm{Ca}^{2+}$ entry by calsequestrin. Sci Rep 2015 ; $5: 11349$.

44. Park H, Park IY, Kim $\varepsilon$, et al. Comparing skeletal and cardiac calsequestrin structures and their calcium binding: a proposed mechanism for coupled calcium binding and protein polymerization. J Biol Chem $2004 ; 279$ : 18026 33.

45. Park H, Wu S, Dunker AK, et al. Polymerization of calsequestrin. Implications for $\mathrm{Ca}^{2+}$ regulation. J Biol Chem $2003 ; 278: 16176-82$.

46. Cho JH, Ko KM, Singaruvelu G, et al. Functional importance of polymerization and localization of calsequestrin in C. elegans. J Cell Sci 2007 ; 120 : 1551-8.

47. Alonso-Jimenez A, Ramon C, Dols-Icardo 0, et al. Corpus callosum agenesis, myopathy and pinpoint pupils: consider Stormorken syndrome. Eur J Neurol $2018 ; 25:$ e $25-6$

48. Shahrizaila N, Lowe J, Wills A. Familial myopathy with tubular aggregates associated with abnormal pupils. Neurology 2004; 63 : 1111-3.

49. Tasca G, D'Amico A, Monforte M, et al. Muscle imaging in patients with tubular aggregate myopathy caused by mutations in STIMl. Neuromuscul Disord $2015 ; 25: 898-903$.

50. Rohkamm R, Boxler K, Ricker K, et al. A dominantly inherited myopathy with excessive tubular aggregates. Neurology 1983 ; 33 : 331-6.

51. Rossi D, Vezzani B, Galli L, et al. A mutation in the CASPl gene causes a vacuolar myopathy with accumulation of sarcoplasmic reticulum protein aggregates. Hum Mutat $2014 ; 35$ : 1163-70.

52. Fahrner M, Stadlbauer M, Muik M, et al. A dual mechanism promotes switching of the Stormorken STIMl R304W mutant into the activated state. Nat Commun $2018 ; 9: 825$

53. Worley PF, Zeng W, Huang GN, et al. TRPC channels as STIMl-regulated storeoperated channels. Cell Calcium $2007 ; 42$ : 205-11.

\section{TIRÉS À PART}

J. Böhm

\section{sfm}

\section{Bulletin d'adhésion 2018}

NOM/Prénom :

$\square$ Clinique $\quad \square$ Fondamentale $\square$ Autre fonction

Adresse :

Code Postal :

Ville :

E-mail :

ADHÉSION : Je désire adhérer en qualité de (rayer la mention inutile)

Membre titulaire : $40 €$

Membre étudiant : gratuit (fournir un justificatif de votre qualité d'étudiant non salarié)

RÈGLEMENT

$\square$ Je joins un chèque libellé à l'ordre de la Société Française de Myologie d'un montant de $40 €$

$\square$ J'effectue un virement bancaire de $40 €$ (RIB de la SFM à demander à Rémi Mounier)

A RETOURNER A :

remi.mounier@univ-lyon1.fr

ou

Rémi MOUNIER - Trésorier de la SFM

CR HDR CNRS - UMR CNRS 5534

Centre de Génétique et de Physiologie Moléculaire et Cellulaire

Université Claude Bernard Lyon 1

Bâtiment Gregor Mendel - 2è étage

16 Rue Raphaël Dubois

F-69622 Villeurbanne Cedex

\section{N.B. : Bulletin à photocopier et à diffuser à toute personne intéressée}

